\title{
Substituição do ligamento redondo por membrana biológica e pino transarticular na redução de luxação coxofemoral bilateral em cães
}

\author{
Substitution of femoral head ligament by biological membrane and \\ transarticular pinnig in reduction of bilateral coxofemoral luxation in dogs \\ Daniel Barbosa Sia ${ }^{1}$, Cristiano Gomes ${ }^{2}$, Emerson Antonio Contesini ${ }^{3}$, Ana Carolina Both ${ }^{4}$, \\ Leandro Haczkiewicz Gaiga ${ }^{4}$, Marcio Poletto Ferreira' ${ }^{2}$, Lucas Marques Colomé5, \\ Eduardo Martins Sousa ${ }^{6}$, Paula Stieven Hünning ${ }^{6}$ \& Suellen Zabalaga Viana ${ }^{6}$
}

\begin{abstract}
RESUMO
A luxação coxofemoral é a mais freqüente nos pequenos animais, sendo geralmente causada por trauma, levando a ruptura do ligamento redondo e da cápsula articular. O exame clínico identifica o tipo de luxação e a severidade da lesão, sendo de fundamental importância também para a avaliação sistêmica do animal traumatizado. O diagnóstico definitivo é realizado através de radiografia simples, que ajuda a direcionar o tratamento. Este trabalho tem o objetivo de relatar a redução de luxação coxofemoral bilateral com ruptura de cápsula e ligamento redondo de um cão. Para tanto, esse ligamento foi substituído por um "flap" de fáscia lata autógena no membro esquerdo e fixada a articulação com pino transarticular no membro direito, na premissa tentativa de redução. Com a reluxação do membro esquerdo, o "flap" de fáscia lata autógena foi substituído por centro frênico eqüino conservado em glicerina $98 \%$. O centro frênico permitiu o uso funcional do membro 15 dias após a cirurgia, enquanto o pino transarticular em apenas 40 dias de pós-operatório. Sugere-se que o centro frênico eqüino conservado possa ser uma alternativa viável na redução de luxação coxofemoral.
\end{abstract}

Descritores: ortopedia, implante, ligamento redondo, luxação coxofemoral, cão.

\section{ABSTRACT}

Coxofemoral luxation is the most common injury in pets. It's generally caused by trauma, creating a rupture in ligament of the femoral head and capsule articulation. The clinical examination identifies the type of luxation and the severity of the injury, being of basic importance also for the systemic valuation of the traumatized animal. The final diagnostic it's from a simple radiographic that help to direct the treatment. The object of this report is to mention a reduction of bilateral coxofemoral luxation where occurred a rupture of capsule and the ligament of the femoral head of a dog. The ligament of the left limb was substituted for autograft fascia lata flap and the right limb had fixed its articulation with a transarticular pinning, in experiment reduction. On left limb reluxation, the autograft fascia lata flap was substituted by equine central tendon preserved in glycerin $98 \%$. The central tendon made possible the functional limb use in 15 days after the operation while the transarticular pinnig just on the 40 days of the postoperative. This report suggest that central tendon, in glycerin conservation, could be a viable alternative on reduction of coxofemoral luxation.

Key words: orthopedics, implant, ligament of the femoral head, coxofemoral luxation, dog. 


\section{INTRODUÇÃO}

As luxações coxofemorais são causadas principalmente após traumatismo externo na pelve e são consideradas as mais comuns em cães [7,17]. $\mathrm{O}$ tratamento inicial para a luxação coxofemoral é a redução fechada e fixação externa com bandagens $[1,3,6$, $7,12]$. A redução aberta é indicada em animais com recidiva da luxação, em luxações com complicações como fraturas e avulsão, ou quando a lesão ocorreu há mais de 5 dias [7].

As reduções abertas apresentam uma taxa de sucesso entre 73 e 100\% [10]. Vários autores descrevem técnicas de redução aberta, as quais estão divididas em extra-capsulares e intracapsulares, incluindo a capsulorrafia e sutura de estabilização extracapsular, a transposição do trocânter maior, a reconstrução capsular por prótese, o pino transarticular, o pino de Toggle ou pino De Vita, o fixador externo flexível, o fio de aço transarticular e os pinos de Steinman inseridos dorsalmente ao acetábulo. Trabalhos atuais demonstram o uso de membranas biológicas como ferramenta para redução de luxações [2,4,5,8,9,11-15,17].

Entretanto, apesar da literatura veterinária descrever algumas técnicas para redução da luxação, em alguns animais pode ocorrer recidiva, restando como opção a artroplastia por excisão da cabeça e colo femorais [8]. Apesar de constituir-se uma opção de tratamento, esta técnica pode culminar com claudicação, pois ocorre encurtamento do membro [4].

O presente trabalho tem como objetivo relatar e avaliar os resultados da redução e estabilização de luxação coxofemoral bilateral através de pino transarticular em um dos membros e de substituição do ligamento redondo por membrana biológica no membro contralateral.

\section{RELATO DE CASO}

Uma cadela da raça poodle, pesando $8,1 \mathrm{~kg}$, com 3 anos de idade e apresentando incapacidade de apoio dos membros posteriores há 1 dia, foi atendida no Hospital de Clínicas Veterinárias (HCV) da Universidade Federal do Rio Grande do Sul (UFRGS). O proprietário relatou na anamnese que o aparecimento do sinal clínico ocorreu devido à queda do animal de seu colo.

Ao exame ortopédico observou-se, nas articulações coxofemorais, crepitação, dor, mobilidade anormal e deslocamento do trocânter maior dorsalmente. Ao exame radiográfico simples, em incidência ventro- dorsal com os membros estendidos, foi constatada a presença de luxação coxofemoral craniodorsal bilateral e um leve arrasamento das fossas acetabulares (Figura 1). Após avaliação clínica específica optouse pela redução e estabilização cirúrgica.

Na medicação pré-anestésica utilizou-se acepromazina $(0,2 \mathrm{mg} / \mathrm{kg})$ associada a meperidina $(2$ $\mathrm{mg} / \mathrm{kg}$ ) por via intramuscular. Em seguida, o paciente foi submetido à tricotomia ampla, estendendo-se da décima terceira costela até a tuberosidade isquiática e da região dorsal dos processos espinhosos até o terço distal da tíbia. A anestesia foi induzida pela administração, de propofol $(5 \mathrm{mg} / \mathrm{kg})$ por via intravenosa, lentamente. Procedeu-se com a intubação orotraqueal com traqueotubo número 7,0 com balonete inflável. A manutenção anestésica contou com o sistema de Bain, utilizando halotano vaporizado e oxigênio a $100 \%$. A profilaxia foi realizada 30 minutos antes do início da cirurgia com ampicilina sódica $(20 \mathrm{mg} / \mathrm{kg})$, por via intravenosa e repetida 2 horas após a primeira aplicação.

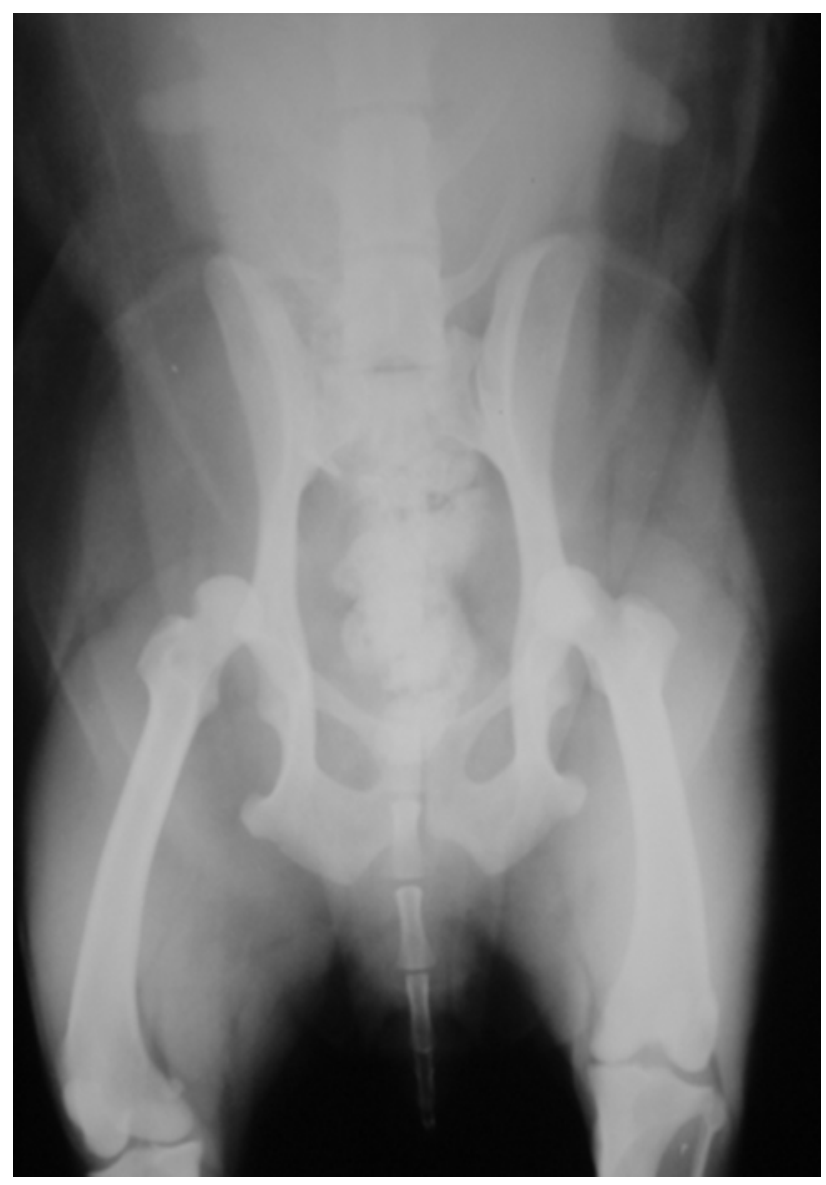

Figura 1. Radigrafia simples em projeção ventrodorsal mostrando luxação coxofemoral craniodorsal bilateral e fossas acetabulares com leve arrasamento. 
O procedimento cirúrgico no membro posterior esquerdo (MPE) iniciou-se com uma incisão de pele a partir do trocânter maior até o côndilo lateral do fêmur para a visualização da fáscia lata. Desta foi confeccionado um retalho, com $1 \mathrm{~cm}$ de largura, do côndilo lateral do fêmur ao músculo tensor da fáscia lata, mantendo-se pediculado a este. A abordagem cirúrgica da articulação foi realizada através de incisão cutânea, iniciando num ponto a meio caminho da diáfise femoral, estendendo-se proximalmente até ultrapassar o trocânter maior e curvando-se cranialmente, para a exposição do músculo glúteo superficial. A incisão continuou dorsalmente, ao longo da margem cranial do músculo glúteo médio, seguida pela secção do músculo glúteo superficial no meio de sua inserção tendinosa. O músculo glúteo médio foi, então, seccionado próximo ao trocânter maior. Os músculos que sofreram tenotomia foram afastados dorsalmente, permitindo a visualização do músculo glúteo profundo e sua secção. A cápsula articular encontrava-se rompida, permitindo a visualização da cabeça femoral, a qual se apresentava deslocada dorsalmente ao acetábulo. Foi realizada a remoção de restos teciduais e de fibrina que se encontravam no acetábulo e na cabeça femoral. Realizou-se um túnel da fovea capitis femoris até o trocânter maior com uma broca de $3,2 \mathrm{~mm}$ e furadeira elétrica (Figura 2) e outro túnel de mesmo diâmetro na fossa acetabular. Um fio de Kirschner de $1 \mathrm{~mm}$ de diâmetro, o qual apresentava uma das extremidades dobrada em forma de alça, foi utilizado para auxiliar na passagem de um fio inabsorvível sintético monofilamentar 2-0 pelo túnel do fêmur, entrando pela fovea capitis femoris e emergindo no trocânter maior (Figura 3). O retalho foi fixado a este fio através de um ponto simples isolado e então tracionado junto com o fio de Kirschner até uma de suas extremidades surgir pela fovea capitis femoris.

Outro fio inabsorvível sintético monofilamentar 2-0 foi fixado a um fio de Kirschner moldado em forma de gancho, o qual foi introduzido pelo túnel acetabular e direcionado, margeando a face pélvica, até surgir na borda dorsal do acetábulo, sendo então retirado, permanecendo apenas o fio inabsorvível sintético monofilamentar, ao qual foi presa a extremidade do retalho que emergiu através da fovea capitis femoris. $\mathrm{O}$ retalho então foi tracionado pelo fio inabsorvível sintético monofilamentar, passando pela borda dorsal do acetábulo (Figura 4). As extremidades do retalho foram fixadas juntamente no trocânter maior com o fio inabsorvível sintético monofilamentar 2-0, com 2 pontos Sultan, após o reposicionamento da cabeça femoral no acetábulo. A cápsula articular não foi suturada devido à laceração que apresentava.

Os músculos glúteos foram suturados às suas inserções, empregando pontos interrompidos de colchoeiro e fio inabsorvível sintético monofilamentar 2-0. O músculo tensor da fáscia lata e a fáscia lata foram suturados com sutura contínua simples e fio poliglactina 910 2-0. A redução de espaço morto em subcutâneo foi realizada com sutura contínua simples e fio poliglactina 910 2-0. A pele foi suturada com ponto simples isolado e fio inabsorvível sintético monofilamentar 3-0.

A abordagem cirúrgica no membro posterior direito (MPD) foi a mesma descrita no procedimento anterior, excetuando-se a incisão para a confecção do retalho de fáscia lata.

Após a exposição da cabeça femoral e a remoção de restos teciduais, reduziu-se a luxação introduzindo um pino de Steinmann de $2 \mathrm{~mm}$ em direção à fovea capitis femoris, a partir do trocânter maior. Assim, com o auxílio de uma parafusadeira elétrica, penetrouse o pino pela fossa acetabular até cerca de $3 \mathrm{~mm}$ no interior da pelve. A rafia das estruturas seguiu o mesmo padrão utilizado no membro contralateral.

No pós-operatório utilizou-se meloxican $(0,1 \mathrm{mg} / \mathrm{kg})$ por via oral, a cada 24 horas, durante 5 dias e enrofloxacina $(5 \mathrm{mg} / \mathrm{kg}$ ) por via oral, a cada 12 horas, durante 7 dias. A limpeza da ferida cirúrgica foi realizada com gaze embebida em solução fisiológica $0,9 \%$, duas vezes ao dia, durante 10 dias.

$\mathrm{O}$ animal foi encaminhado para casa sob as recomendações do pós-operatório prescritas. O proprietário, neste momento, foi instruído a respeito do preenchimento do formulário, para a observação diária de deambulação do paciente, conforme a graduação de claudicação citada por Tudury e Raiser [18] (Tabela 1). Após 10 dias o paciente foi avaliado, sendo observado, ao exame clínico do MPD, movimentação de flexão e extensão normais, abdução e adução restritas e leve dor à palpação. Já o MPE apresentava crepitação, mobilidade anormal, dor e deslocamento dorsal do terceiro trocânter. Segundo formulário de deambulação preenchido pelo proprietário, verificou-se, no $8^{\circ}$ dia, o retorno da claudicação intensa do MPE. Ao exame radiográfico confirmou-se a recidiva da luxação coxofemoral do MPE. 
Tabela 1. Características clínicas dos cinco graus utilizados para avaliar a recuperação do uso funcional do membro, após redução da luxação coxofemoral.

\begin{tabular}{cl}
\hline Grau & \multicolumn{1}{c}{ CARACTERÍSTICAS } \\
\hline I & $\begin{array}{l}\text { Não usa nem apóia o membro. } \\
\text { Uso e apoio infreqüentes do membro na estação } \\
\text { e no caminhar. Não suporta peso na extremidade } \\
\text { afetada, elevando-a ao correr. } \\
\text { Claudicante uso do membro na estação } \\
\text { III no caminhar. Parcial suporte do peso } \\
\text { na extremidade, elevando-a ao correr. }\end{array}$ \\
IV $\quad \begin{array}{l}\text { Caminhar sem claudicar. Normal na estação. } \\
\text { Claudica ao correr sem elevar o membro. }\end{array}$ \\
V $\quad$\begin{tabular}{l} 
Uso funcional do membro. \\
\hline
\end{tabular}
\end{tabular}

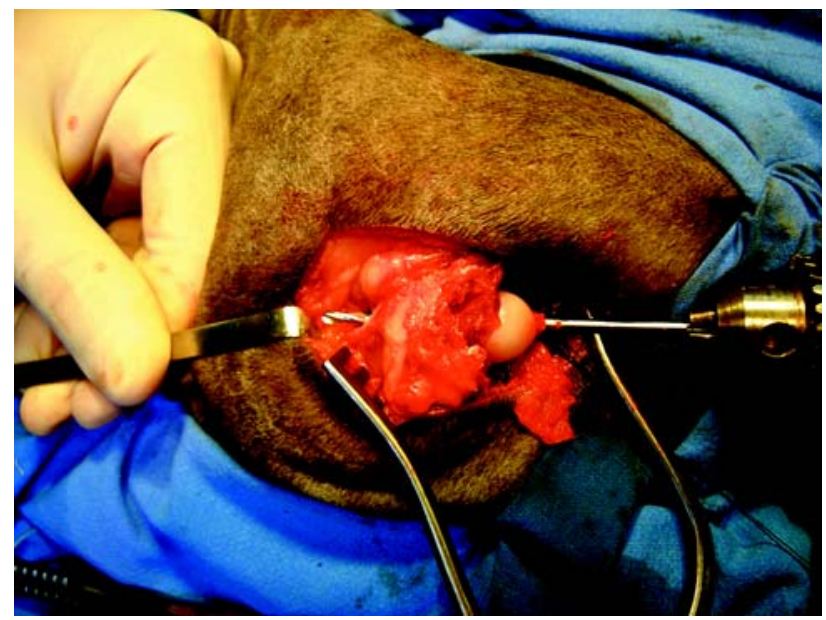

Figura 2. Realização do túnel no fêmur com uma broca de $3,2 \mathrm{~mm}$ e furadeira elétrica. Notar a entrada da broca pela fovea capitis e saída no trocânter maior.

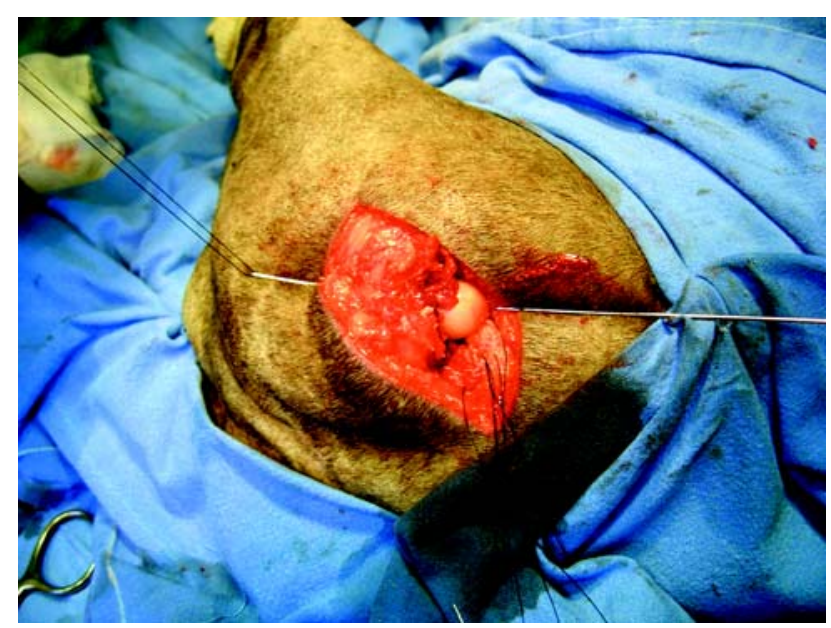

Figura 3. Passagem do fio de Kirschner de $1 \mathrm{~mm}$ de diâmetro através do túnel femoral, entrando pela fovea capitis e emergindo no trocânter maior. Notar a fixação do fio de mononylon 2-0 à extremidade emergente do fio de Kirschner.

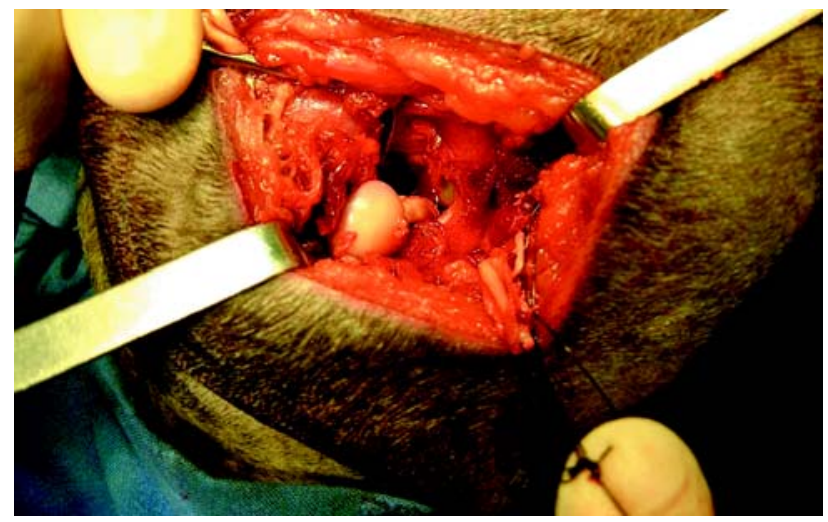

Figura 4. Autoenxerto de fáscia lata passando pelo trocanter maior, fovea capitis e fossa acetabular e emergindo na porção dorsal do acetábulo.

O paciente foi encaminhado à cirurgia para redução e estabilização da articulação, sendo submetido aos mesmos procedimentos pré-cirúrgicos e cirúrgicos anteriormente relatados. Entretanto, em substituição ao retalho de fáscia lata, foi utilizado implante de centro frênico eqüino ( $1 \mathrm{~cm}$ de largura e $20 \mathrm{~cm}$ de comprimento) conservado em glicerina $98 \%$ durante 30 dias.

O pós-operatório foi o mesmo do primeiro procedimento, porém o animal permaneceu internado em canil de $1 \mathrm{~m}^{2}$ no HCV/UFRGS durante 7 dias, sendo levado a passeio sob observação 2 vezes ao dia. Após esse período, recebeu alta e retornou ao seu ambiente de origem com recomendação de repouso moderado por mais 15 dias.

$\mathrm{O}$ animal foi acompanhado por mais 60 dias com realização de exames clínicos semanais e exames radiográficos aos 30 e 60 dias pós-cirurgia.

O pino transarticular foi retirado no $30^{\circ}$ dia com o animal sob anestesia geral, através de incisão sobre o terceiro trocânter e divulsão do subcutâneo e da musculatura até a visualização do pino para a sua remoção com alicate de pressão.

\section{DISCUSSÃO}

Através do acompanhamento periódico do paciente com a realização de exames clínicos e radiográficos e através do preenchimento do formulário pelo proprietário, pode-se obter resultados sobre a evolução do caso.

Os resultados apresentados em relação à deambulação (Tabela 2) diferem dos obtidos por Moya [13] no "loop" pediculado de fáscia lata autógena, principalmente, pela ausência de repouso em gaiola durante 
Tabela 2. Evolução do grau de claudicação em relação às técnicas empregadas.

\begin{tabular}{lccccccc}
\hline & $\begin{array}{c}\text { Período de } \\
\text { observação }\end{array}$ & \multicolumn{5}{c}{ Grau de claudicação/dias } \\
\cline { 3 - 8 } & & I & II & III & IV & V \\
\hline MPD (pino) & 10 dias & 1 & 3 & 5 & 10 & 40 \\
MPE (fáscia lata) & 60 dias & 1 & 5 & 7 & 9 & 15 \\
MPE (centro frênico) & Representa o retorno ao grau I de claudicação no & $8^{\circ}$ & dia. & & & &
\end{tabular}

membranas podemos citar o emprego da fáscia lata em substituição ao ligamento cruzado cranial [16] ou em substituição ao ligamento redondo da cabeça femoral $[2,10]$, o emprego de centro frênico na reparação de lesões tendíneas em coelhos [15], o uso de pericárdio canino na reparação de lesões musculares em ratos Wistar [5] e aplicação de pericárdio bovino em artroplastia acetábulo-femoral em cães [14].

A ausência de reluxação, o retorno precoce à função locomotora normal do membro posterior esquerdo, a visível diminuição de dor no pós-operatório,

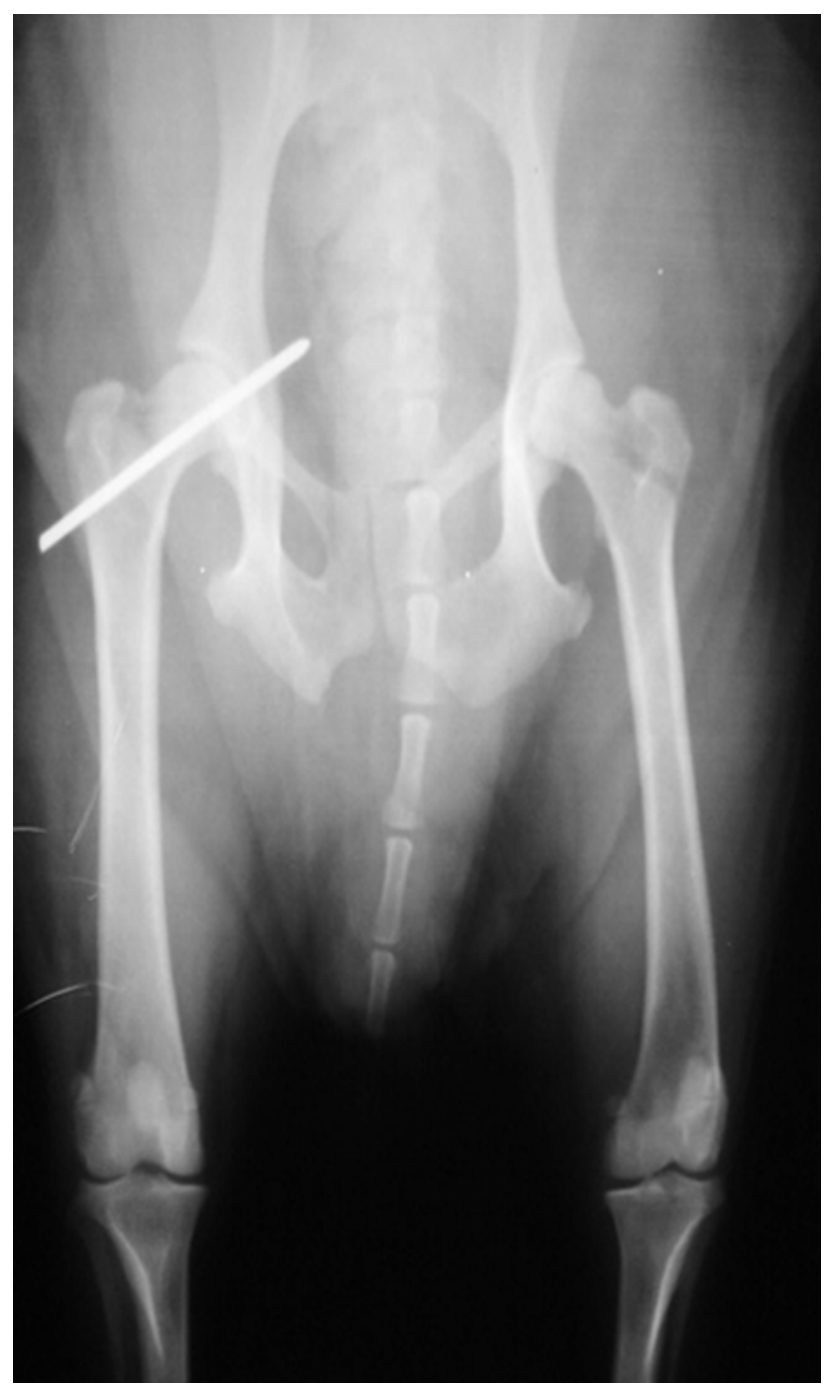

Figura 5. Radiografia simples em projeção ventrodorsal mostrando articulação coxofemoral direita congruente e estabilizada com pino de Steinmann transarticular e articulação coxofemoral esquerda congruente e com túnel mais radioluscente no colo e cabeça femorais condizente à perfuração para passagem do autoenxerto de fáscia lata. 
Sia D.B., Gomes C., Contesini E.A., Both A.C., Gaiga L.H., Ferreira M.P., Colomé L.M., Sousa E.M., Hünning P.S. \& Viana S.Z. 2006. Substituição do ligamento redondo por membrana biológica...

Acta Scientiae Veterinariae. 34: 187-192.

a movimentação fisiológica da articulação e a não necessidade de intervenção cirúrgica posterior, relatados neste trabalho, comprovaram a eficácia e as vantagens da substituição do ligamento redondo por centro frênico equino para a estabilização da articulação coxofemoral em cães.

\section{REFERÊNCIAS}

1 Bone D.L., Walker M. \& Cantewell H.D. 1984. Traumatic coxofemoral in dogs: results of repair. Veterinary Surgery. 13: 263-270.

2 Brandão C.V.S., Iamaguti P. \& Figueiredo L.M.A. 2000. Substituição do ligamento da cabeça do fêmur com autoenxerto de fáscia lata na luxação coxofemoral em cães. Ciência Rural. 30: 275-280.

3 Brasher A.W.P., Walter M.C. \& Newton C.D. 1986. Coxofemoral luxation in the dog and cat. Veterinary Surgery. 15: $356-362$.

4 Brinker W.O., Piermattei D.L. \& Flo G.L. 1986. Diagnóstico e tratamento dos estados ortopédicos do membro posterior. In: Manual de ortopedia e tratamento das fraturas dos pequenos animais. São Paulo: Manole, pp.282-292.

5 Brun M.V., Pippi N.L., Dreimeier D., Contesini E.A., Beck C.A.C., Cunha O., Pinto F.S.T.L., Roeshig C. \& Stedile R. 2002. Solução hipersaturada de sal como conservante de pericárdio canino utilizado na reparação do músculo reto abdominal de ratos Wistar. Ciência Rural. 32: 1019-1025.

6 Dallmann M.J. \& Mann F.A. 1981. Reduction and management of coxofemoral luxations in the dog and cat. Journal of Veterinary Orthopedics. 3: 7-13.

7 Evers P., Johnston G.R., Wallace L.J., Lipowitz A.J. \& King V.L. 1997. Long-term results of treatment of traumatic coxofemoral joint dislocation in dogs: 64 cases. Journal of American Veterinary Medical Association. 210: 59-64.

8 Fossum T.W. 1997. Management of joint diseases. In: Small Animal Sugery. Sant Louis: Mosby, pp.883-998.

9 Goelzer L.P. 2001. Estabilização coxofemoral extra-articular após redução de luxação craniodorsal em cães. 33 f. Santa Maria, RS. Dissertação (Mestrado em Ciências Veterinárias) - Programa de Pós-graduação em Ciências Veterinárias, Universidade Federal de Santa Maria.

10 Hammer D.L. 1980. Recurrent coxofemoral luxation in fifteen dogs and one cat. Journal of the American Veterinary Medical Association. 177: 1018-1020.

11 Kawamata T., Niiyama M. \& Taniyama H. 1996. Open reduction and stabilisation of coxofemoral join luxation in dogs and cats, using a stainless steel rope iserted via a ventral approach to the hip joint. Australian Veterinary Journal. 74: 460-463.

12 Mclaughlin R.E. 1995. Traumatic joint luxation in small animals. Veterinary Clinics of North American Small Animal Practice. 25: $1175-1196$.

13 Moya L.E.G. 2001. Substituição do ligamento Redondo por "loop" pediculado de fáscia lata em cães. 42f. Santa Maria, RS. Dissertação (Mestrado em Ciências Veterinárias) - Programa de Pós-graduação em Ciências Veterinárias, Universidade Federal de Santa Maria.

14 Rodaski S., Cunha O., De Nardi,A.B., Rios A., Comar F.A. \& Castro J.H.T. 2002. Artroplastia acetábulo-femoral em cães com pericárdio bovino conservado. Archives of Veterinary Science. 7: 179-187.

15 Sartor Filho R., Gandolfi W. \& Bandarra E.P. 1997. Emprego de membrana biológica (centro frênico) na reparação das lesões tendíneas em coelhos. Veterinária e Zootecnia. 9: 69-77.

16 Silva A.M., Carlo R.J.D. \& Fonseca C.C. 2002. Aspectos macro e microscópicos da fáscia lata utilizada como substituto autógeno do ligamento cruzado cranial. Ciência Rural. 30: 275-280.

17 Tomlinson J.L. 1996. Redução das Luxações Coxofemorais. In: Bojrab M.J. (Ed.) Técnicas Atuais de Cirurgia de Pequenos Animais. 3.ed. São Paulo: Roca, pp.622-691.

18 Tudury E.A. \& Raiser A.G. 1985. Redução de fraturas distais de fêmur de cães empregando dois pinos de Steinmann em substituição aos de Rush. Revista do Centro de Ciências Rurais. 15: 141-155.

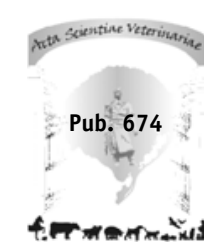

